\title{
Nesfatin 1: a promising biomarker predicting successful reperfusion after coronary artery bypass surgery
}

\author{
Kirisci $\mathrm{M}^{1}$, Yardimci $\mathrm{MM}^{2}$, Kocarslan $\mathrm{A}^{1}$, Sokmen $\mathrm{A}^{3}$, Doganer $\mathrm{A}^{4}$, Gunes $\mathrm{H}^{3}$ \\ Kahramanmaras Sütcü Imam University, Faculty of Medicine, Department of Cardiovascular Surgery \\ Kahramanmaras, Turkey. drkirisci@yahoo.com
}

\begin{abstract}
OBJECTIVES: Nesfatin-1 is an antiiflammatory, antiapoptotic, and anorexigenic peptide that has many roles in cardiomyocyte metabolism and viability. Inflammation plays an important role in the pathogenesis of atherosclerosis. In this study, we aimed to evaluate the alterations in serum nesfatin-1 levels of the patients undergoing coronary artery bypass operation due to atherosclerotic coronary artery disease.

MATERIALS AND METHODS: The study included 49 patients (30 men, 19 women) undergoing coronary artery bypass surgery. Serum nesfatin-1 levels were measured from venous blood samples of the patients collected before and three months after the operation. The relationship of nesfatin-1 levels with accompanying conditions was also analyzed.

RESULTS: Nesfatin-1 levels at third month, postoperatively, were significantly higher than preoperative nesfatin-1 levels of the patients $(41.94 \pm 13.90 \mathrm{pg} / \mathrm{ml}$ and $27.06 \pm 8.01 \mathrm{pg} / \mathrm{ml}$, respectively; $p<0.001)$. Both preoperative and postoperative nesfatin- 1 levels were negatively correlated with age $(p<0.001)$. The postoperative increase in nesfatin-1 levels was significantly higher in diabetic patients than in nondiabetic patients $(p<0.001)$.

CONCLUSION: This study revealed that serum nesfatin-1 levels increased significantly in patients undergoing coronary artery bypass operation. Nesfatin- 1 level may have a role in assessing myocardial perfusion during the follow-up of these patients (Tab. 4, Fig. 4, Ref. 25). Text in PDF www.elis.sk.

KEY WORDS: atherosclerosis, coronary bypass, inflammation, nesfatin-1.
\end{abstract}

\section{Introduction}

Nesfatin-1 was first described by Oh-I and colleagues in 2006 as an anorexigenic neuropeptide originating from nucleobindin-2 (NUCB2) which is secreted by the hypothalamus (1). Nesfatin-1 has been shown to be present in subcutaneous fat tissue, pancreatic endocrine cells, testis, skeletal muscle, hypophysis gland, liver, sympathetic nervous system, and peripheral tissues like sacral parasympathetic preganglionic neurons in the spinal cord (2-4). Nesfatin-1 improves glucose and lipid metabolism by increasing the use of free fatty acids (5). It has been shown in a study conducted in traumatic rat brains that nesfatin- 1 has anti-inflammatory and antiapoptotic effects (6). Plasma nesfatin-1 levels have been shown to increase in patients with epilepsy or major depression $(7,8)$.

${ }^{1}$ Kahramanmaras Sütcü Imam University, Faculty of Medicine, Department of Cardiovascular Surgery Kahramanmaras, Turkey, ${ }^{2}$ Sanko University, Faculty of Medicine, Department of Cardiovascular Surgery Gaziantep, Turkey, ${ }^{3}$ Kahramanmaras Sütcü İmam University, Faculty of Medicine, Department of Cardiology Kahramanmaras, Turkey, and ${ }^{4}$ Kahramanmaras Sütcü İmam University, Faculty of Medicine, Department of Biostatistics Kahramanmaras, Turkey

Address for correspondence: M. Kirisci Mehmet, MD, Kahramanmaras Sütcü İmam University, Faculty of Medicine, Department of Cardiovascular Surgery Kahramanmaras, Turkey.

Phone: +905057181898, Fax: +903443003409
It has been reported that plasma nesfatin-1 levels in patients with slow coronary flow were lower with regard to the subjects having normal coronary flow (9). Nesfatin-1 has many roles in cardiac pathophysiology, such as regulating blood pressure, heart rate, cardiomyocyte metabolism and viability (10).

Cardiovascular diseases are the leading cause of death worldwide (11). The term of cardiovascular disease (CVD) is used for all of the diseases including coronary artery disease (CAD), myocardial infarction (MI), heart failure (HF), cardiomyopathy, arrhythmia, valvular heart disease, stroke, and atherosclerosis $(12,13)$. CAD is the most frequently seen disease among them (11). The main etiology in the pathogenesis of CAD is the development of agerelated atherosclerosis, while chronic vascular inflammation plays a significant role in the etiopathogenesis of atherosclerosis $(14,15)$.

In this study, we aimed to evaluate the differences between preoperative and postoperative serum nesfatin-1levels in patients undergoing coronary artery bypass operation due to atherosclerotic CAD. We also evaluated the alterations in serum nesfatin-1 levels based on demographic data of the patients.

\section{Methods}

\section{Study population}

The study included 52 patients undergoing coronary artery bypass operation at the department of cardiovascular surgery 
Tab. 1. Clinical characteristics of the study population.

\begin{tabular}{lcc}
\hline Age (years) $($ mean \pm SD) & & $62.92 \pm 9.48$ \\
\hline \multirow{2}{*}{ Sex n (\%) } & Male & $30(61.22)$ \\
& Female & $19(38.78)$ \\
\hline \multirow{2}{*}{ Smoking n (\%) } & No & $40(81.63)$ \\
\hline \multirow{2}{*}{ Hypertension n (\%) } & Yes & $9(18.37)$ \\
\hline \multirow{2}{*}{ Hyperlipidemia n (\%) } & No & $15(30.61)$ \\
& Yes & $34(69.39)$ \\
\hline \multirow{2}{*}{ Family history n (\%) } & No & $34(69.39)$ \\
& Yes & $15(30.61)$ \\
\multirow{2}{*}{ DM n (\%) } & No & $16(32.65)$ \\
\multirow{2}{*}{ COPD n (\%) } & Yes & $33(67.35)$ \\
\hline \multirow{2}{*}{ Additional comorbidity n (\%) } & No & $24(48.98)$ \\
& Yes & $25(51.02)$ \\
\hline
\end{tabular}

COPD - chronic obstructive pulmonary disease, DM - diabetes mellitus

Tab. 2. Operative data of the study patients.

\begin{tabular}{|c|c|c|c|}
\hline Bypass number & Median(Q1-Q3) & \multicolumn{2}{|c|}{$3(3.00-4.00)$} \\
\hline $\begin{array}{l}\text { Duration of hospitalization } \\
\text { (days) }\end{array}$ & Median(Q1-Q3) & \multicolumn{2}{|c|}{$8(8.00-9.00)$} \\
\hline $\begin{array}{l}\text { Duration of intensive care } \\
\text { (days) }\end{array}$ & Median(Q1-Q3) & \multicolumn{2}{|c|}{$2(1.00-2.00)$} \\
\hline Mortality & $\begin{array}{l}\text { No } \\
\text { Yes }\end{array}$ & $\begin{array}{l}\mathrm{n}(\%) \\
\mathrm{n}(\%)\end{array}$ & $\begin{array}{c}49(100.00) \\
0(0.00)\end{array}$ \\
\hline LIMA nesfatin-1 staining & $\begin{array}{l}\text { No } \\
\text { Yes }\end{array}$ & $\begin{array}{l}\mathrm{n}(\%) \\
\mathrm{n}(\%)\end{array}$ & $\begin{array}{l}16(38.10) \\
26(61.90) \\
\end{array}$ \\
\hline
\end{tabular}

LIMA - Left internal mammary artery

Tab. 3. Preoperative and postoperative serum nesfatin-1 levels.

\begin{tabular}{lccc}
\hline & $\begin{array}{c}\text { Preoperative } \\
(\text { mean } \pm \text { SD) }\end{array}$ & $\begin{array}{c}\text { Postoperative } \\
(\text { mean } \pm \text { SD) }\end{array}$ & $\mathrm{p}$ \\
\hline $\begin{array}{l}\text { Nesfatin-1 } \\
(\mathrm{n}: 49)(\mathrm{pg} / \mathrm{ml})\end{array}$ & $27.06 \pm 8.01$ & $41.94 \pm 13.90$ & $\mathrm{p}<0.001 *$ \\
\hline Paired sample t-test; $a-0.05 * *$ difference is statistically significant &
\end{tabular}

of Kahramanmaras Sutcu Imam University Research Hospital between October 2017 and July 2019. The patients underwent clinical, electrocardiographic, and echocardiographic evaluations at 3rd month of postoperative follow-up while 3 patients determined to have ischemia confirmed by a cardiologist as a result of this evaluation were excluded from the study. In total, 49 patients (30 men, 19 women; mean age: $62.92 \pm 9.48$ years) without signs of ischemia 3 months after the operation were included in the study. Venous blood samples were taken from the patients before and 3 months after the operation. The control blood samples were planned to be taken 3 months after the operation in order to avoid the effect of inflammatory response caused by the operation. Serum nesfatin-1 levels were measured from collected blood samples. The demographic data of all patients were recorded. The patients with acute myocardial infarction, congenital heart disease, cardiomyopathy, valvular heart disease, congestive heart failure, malignancy, infectious diseases, acute or chronic inflammatory diseases, psychiatric diseases, neurological diseases, hematological diseases, renal failure, liver failure, hematological diseases, and those with the history of operation or trauma in the past one month were excluded from the study.

The study was approved by the Institutional Ethics Committee (approval date: September 27, 2017; document number 6). Before participating in the study, informed written consent was obtained from each subject after explaining the protocol.

\section{Laboratory measurements}

Venous blood samples were collected from antecubital fossa into the glass tubes containing ethylenediaminetetraacetic acid (EDTA) preoperatively and in the morning, one month after the operation. Serum and plasma were separated and stored at $-80^{\circ} \mathrm{C}$ for the analysis of nesfatin-1. Serum nesfatin-1 levels were measured using a commercial enzyme-linked immunosorbent assay (ELISA) kit (sensitivity: $<10$ pg/ml; assay range: $31.2-2000$ pg/ml; Boster Immunoleader, USA) as recommended by the manufacturer's protocol.

\section{Statistical analysis}

Shapiro-Wilk test was used to test the normality of distribution for continuous variables. Mean values of continuous variables were compared between preoperative and postoperative groups using paired sample t-test or Wilcoxon test according to whether normally distributed or not. The relationship between quantitative variables was examined by Pearson correlation test. Statistical parameters were expressed as means \pm standard deviation and median ( $25 \%$ quartile - $75 \%$ quartile). Data were analyzed with IBM SPSS version 22 (IBM SPSS for Windows version 22, IBM Corporation, Armonk, New York, United States). $\mathrm{p}<0.05$ was accepted as statistically significant.

\section{Results}

Clinical characteristics and operative data of the patients undergoing coronary artery bypass operation are given in Table 1 and Table 2, respectively. Nesfatin-1 levels assessed one month after surgery were found to be increased significantly as compared to preoperative nesfatin- 1 levels $(41.94 \pm 13.90 \mathrm{pg} / \mathrm{ml}$ vs $27.06 \pm 8.01$ $\mathrm{pg} / \mathrm{ml} ; \mathrm{p}<0.001$ ) (Tab. 3, Fig. 1).

The differences between preoperative and postoperative nesfatin-1 levels are shown in Table 4 according to the clinical fea-

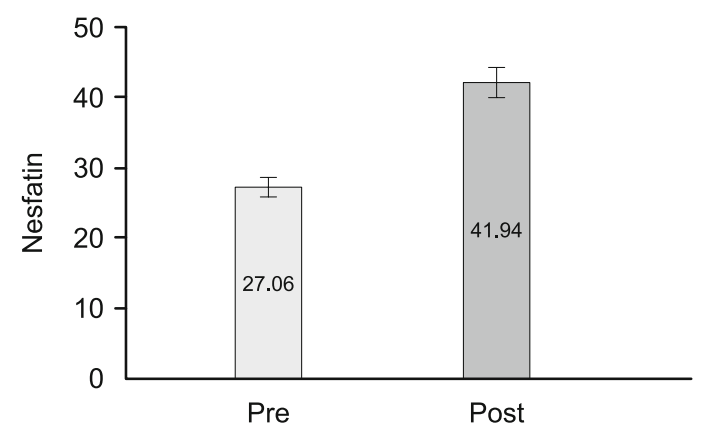

Fig. 1. Preoperative and postoperative serum nesfatin-1 levels. 
Tab. 4. The relationship of nesfatin-1 levels with demographic data.

\begin{tabular}{|c|c|c|c|c|c|}
\hline & & \multicolumn{2}{|c|}{ Nesfatin-1 (Mean \pm SD $)$} & \multirow[b]{2}{*}{$\mathrm{t} / \mathrm{Za}$} & \multirow[b]{2}{*}{$\mathrm{p}$} \\
\hline & & Preoperative & Postoperative & & \\
\hline \multirow{2}{*}{ Sex } & Male & $27.83 \pm 8.35$ & $43.43 \pm 14.36$ & 8.336 & $\mathrm{p}<0.001^{*}$ \\
\hline & Female & $25.84 \pm 7.51$ & $39.58 \pm 13.19$ & 5.254 & $\mathrm{p}<0.001^{*}$ \\
\hline \multirow{2}{*}{ Smoking } & No & $26.63 \pm 7.81$ & $41.57 \pm 14.09$ & 8.352 & $\mathrm{p}<0.001^{*}$ \\
\hline & Yes & $29.00 \pm 9.11$ & $43.56 \pm 13.74$ & 5.982 & $\mathrm{p}<0.001^{*}$ \\
\hline \multirow{2}{*}{ Hypertension } & No & $25.80 \pm 8.51$ & $39.60 \pm 14.76$ & 5.005 & $\mathrm{p}<0.001^{*}$ \\
\hline & Yes & $27.62 \pm 7.85$ & $42.97 \pm 13.61$ & 8.338 & $\mathrm{p}<0.001^{*}$ \\
\hline \multirow{2}{*}{ Hyperlipidemia } & No & $26.50 \pm 8.30$ & $39.82 \pm 13.44$ & 7.616 & $\mathrm{p}<0.001^{*}$ \\
\hline & Yes & $28.33 \pm 7.44$ & $46.73 \pm 14.21$ & 6.423 & $\mathrm{p}<0.001^{*}$ \\
\hline \multirow{2}{*}{ Family history } & No & $24.12 \pm 6.76$ & $38.81 \pm 13.78$ & 6.537 & $\mathrm{p}<0.001^{*}$ \\
\hline & Yes & $28.48 \pm 8.28$ & $43.45 \pm 13.92$ & 7.495 & $\mathrm{p}<0.001 *$ \\
\hline \multirow{2}{*}{ DM } & No & $25.75 \pm 7.95$ & $36.71 \pm 10.61$ & 6.663 & $\mathrm{p}<0.001^{*}$ \\
\hline & Yes & $28.32 \pm 8.04$ & $46.96 \pm 15.01$ & 8.059 & $\mathrm{p}<0.001^{*}$ \\
\hline \multirow[t]{2}{*}{ LIMA nesfatin1 staining } & No & $26.13 \pm 6.40$ & $41.19 \pm 14.94$ & 4.778 & $\mathrm{p}<0.001^{*}$ \\
\hline & Yes & $27.27 \pm 8.56$ & $42.04 \pm 14.30$ & 8.319 & $\mathrm{p}<0.001^{*}$ \\
\hline
\end{tabular}

a Wilcoxon test; paired sample t-test; a-0,05; *difference of preoperative-postoperative is statistically significant

caused by atherosclerosis, namely patients with carotid artery stenosis less than $60 \%$, patients with carotid artery stenosis more than $60 \%$ and patients with normal carotid artery. They found a negative correlation between serum nesfatin-1 level and severity of carotid artery stenosis. In this study, serum nesfatin-1 level of patients with carotid artery stenosis $>60 \%$ was found to be significantly lower as compared to other groups. In addition, the nesfatin-1 level of patients with carotid artery stenosis $<60 \%$ was also found to be significantly lower as compared to patients with normal carotid arteries (20). Shimei et al compared nesfatin-1 levels of diabetic patients with and without peripheral artery disease and found that serum nesfatin-1 levels were signifi-

tures such as sex, smoking, hypertension, hyperlipidemia, family history, diabetes mellitus, and staining of left internal mammary artery (LIMA). Nesfatin-1 levels increased significantly at the end of third month with regard to preoperative nesfatin-1 levels, independently from clinical parameters $(\mathrm{p}<0.001)$.

Preoperative and postoperative nesfatin-1 level was found to be negatively correlated with age. Although serum nesfatin-1 level increased postoperatively in all patients, the increase rate was lower in older patients $(\mathrm{p}<0.001)$ (Fig 2 and 3$)$.

When postoperative nesfatin-1 levels of patients with and without DM were compared, it was seen that the postoperative increase rate of nesfatin-1 level was significantly higher in patients with DM (p<0.001) (Fig. 4).

\section{Discussion}

To the best of our knowledge, this is the first study investigating the relationship between nesfatin-1 and coronary artery bypass grafting, and it has been shown that postoperative serum nesfatin-1 level has increased three months after the operation independently of other risk factors.

Nesfatin-1 is primarily recognized as a satiety hormone (1). The secretion of adipocytokines such as nesfatin-1 from adipose tissue has been shown to have an effect on cardiovascular diseases (16). Atherosclerosis has an important role in the development of cardiovascular diseases (17). Inflammation plays a role in every stage of atherosclerosis in formation, progression and rupture of atherosclerotic plaques (18). Tang et al have shown the antiinflammatory and antiapoptotic effects of nesfatin-1 molecule in rats with subarachnoid hemorrhage (6). Dai et al compared serum nesfatin-1 levels of patients with acute MI, stable angina pectoris and normal coronary arteries with each other. They found that serum nesfatin-1 level of patients with acute MI was significantly lower as compared to other two groups, and suggested that lower nesfatin-1 level might play an important role in the development of acute MI (19). Kuyumcu et al investigated serum nesfatin-1 level in three groups of patients with carotid artery stenosis cantly lower in patients with peripheral artery disease. Based on the results of the study, they suggested that nesfatin-1 might be a biomarker to predict the development of peripheral artery disease caused by atherosclerosis (21). Tasatargil et al induced MI in rats by isoproterenol infusion, and investigated serum troponin- $\mathrm{T}$ level and levels of inflammatory markers such as interleukin-6 (IL-6), tumor necrotizing factor Alfa (TNF- $\alpha$ ), and interleukin-1 beta (IL-1 $\beta$ ) after an administration of intraperitoneal nesfatin-1. Myocardial tissue samples were also analyzed microscopically with regard to apoptosis and necrosis. Serum troponin-T, IL-6, TNF- $\alpha$ and IL- $1 \beta$ values were found to be significantly lower in rats administered with nesfatin-1. Similarly, apoptotic and necrotic cells caused by MI were shown to be decreased in rats administered with nesfatin-1. Based on these results of the study, they claimed nesfatin-1 to have a cardioprotective effect (22). In our study, we showed that preoperative nesfatin-1 levels of patients undergoing a coronary artery bypass operation increased significantly one month after the operation. Previous studies reported a negative correlation between nesfatin-1 level and the

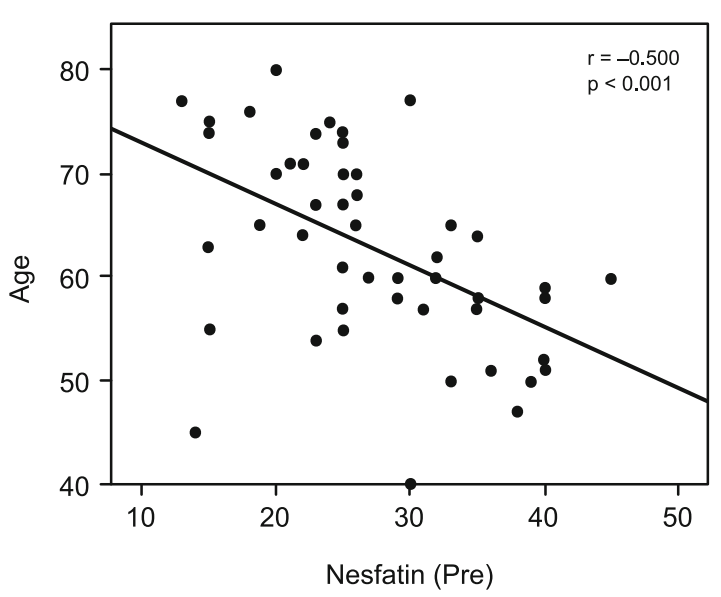

Fig. 2. Correlation of preoperative nesfatin-1 level with age. 


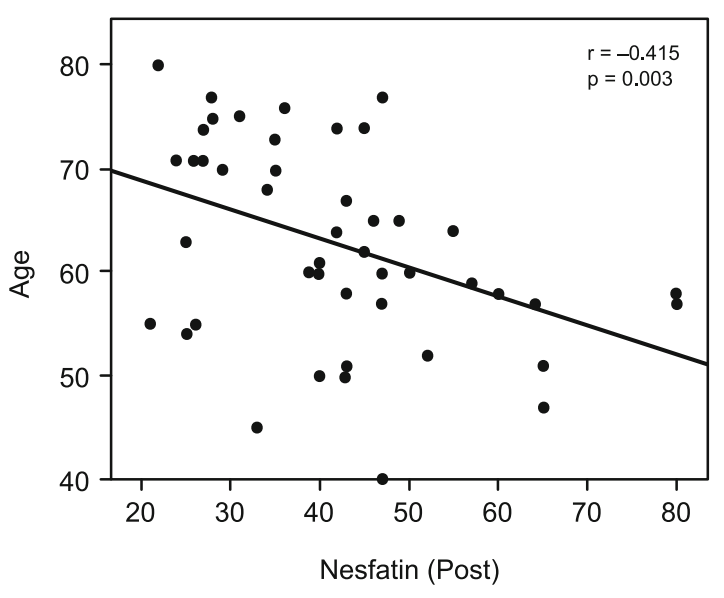

Fig. 3. Correlation of postoperative nesfatin-1 level with age.

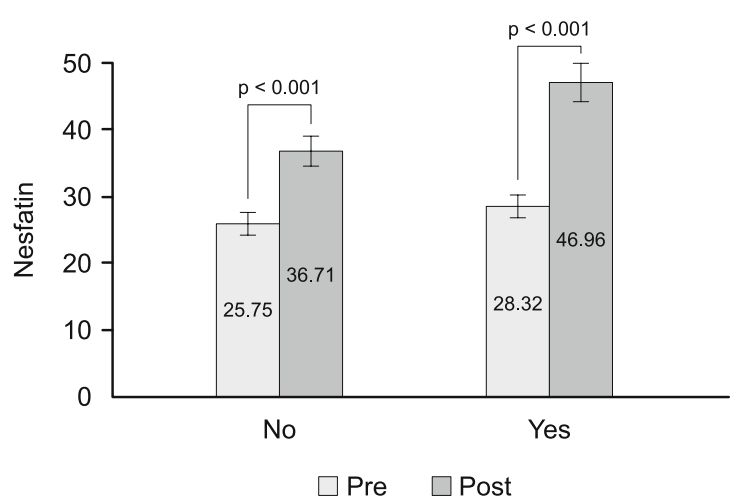

Fig. 4. Relationship of nesfatin-1 level with DM.

severity of atherosclerotic vascular diseases. Similarly, decreased serum nesfatin-1 level in atherosclerotic coronary artery disease was shown to be increased after coronary artery bypass operation in our study. This may be explained by restoration of coronary circulation and reversal of ischemia after coronary bypass operation. The increase in nesfatin-1 level after coronary artery bypass operation may be a marker indicating a cardioprotective effect of revascularization and can be used for follow-up of these patients postoperatively.

When we analyzed postoperative nesfatin-1 levels in terms of hypertension, DM, sex, smoking and family history it was seen that postoperative nesfatin-1 levels increased independently from all these risk factors. It was seen upon the comparison of patients with and without staining of LIMA by tissue dye of nesfatin-1 that postoperative nesfatin-1 levels were also significantly increased in both groups of patients. These results show that postoperative increase in nesfatin-1 levels may be an independent indicator of improvement in coronary circulation.

In this study, nesfatin-1 level was shown to be negatively correlated with age. Nesfatin-1 level increased postoperatively in all age groups, but preoperative and postoperative nesfatin-1 levels in the older group were lower as compared to younger patients. In contrast to our study, Li et al showed a positive correlation between age and nesfatin-1 level (23). There is a paucity of data in the literature about the relationship between age and nesfatin-1 level. It is well known that aging is an important factor in the progression of atherosclerosis, therefore we suggest that nesfatin-1 level may be expected to be lower in elderly patients.

When we compared the alteration of nesfatin-1 level postoperatively in patients with and without DM, the postoperative increase rate of nesfatin-1 in patients with DM was found to be higher. Dai et al compared nesfatin-1 levels of the diabetic patients having MI with those of the non-diabetic patients having MI in their study and found no significant difference in serum nesfatin-1 levels between those groups (19). Li et al studied fasting nesfatin-1 levels in three groups of patients, namely those with type 1 DM, type 2 DM, and healthy subjects. They revealed that serum nesfatin-1 levels in patients with type $2 \mathrm{DM}$ were significantly lower than those in other groups (23). Zai et al published a metaanalysis of seven studies conducted between the years of 2010 and 2017, which investigated the relationship between nesfatin-1 and DM (24). In one of these studies, nesfatin-1 was claimed to exert an antihyperglycemic effect, and it was shown that antidiabetic agents decreased the serum nesfatin-1 level as a result of the decreased glucose level (25). In their meta analysis Zai et al reported that there might be a relationship between nesfatin-1 level and DM, and that further studies were needed to document this relationship clearly. In our study, the preoperative nesfatin-1 levels in patients with and without DM were similar. However, the postoperative increase rate of nesfatin-1 level was seen to be higher in patients with DM. According to these results, the higher increase in postoperative nesfatin-1 levels in patients with DM may predict that diabetic patients may benefit more from bypass surgery. Further studies with larger study population are needed to clarify this subject.

\section{Conclusion}

In conclusion, this study revealed that serum nesfatin-1 levels increased significantly in patients undergoing coronary artery bypass operation. Nesfatin-1 level may have a role in assessing myocardial perfusion during the follow-up of these patients. Further studies are needed to clarify the relationship of nesfatin-1 with coronary artery revascularization.

\section{References}

1. Ohi S, Shimizu H, Satoh T et al. Identification of nesfatin-1 as a satiety molecule in the hypothalamus. Nature 2006; 443 (7112):. 709-712.

2. Ayada C, Toru U, Korkut Y. Nesfatin-1 and its effects on different systems. Hippokratia 2015; 19: 4-10.

3. Ramanjaneya M,Chen J, Brown JE et al. Identification of nesfatin-1 in human and murine adipose tissue: a novel depot-specific adipokine with increased levels in obesity. Endocrinology 2010; 151: 3169-3180.

4. Foo KS, Brauner H, Ostenson CG, Broberger C. Nucleobindin-2/ nesfatin in the endocrine pancreas: distribution and relationship to glycaemic state. J Endocrinol 2010; 204: 255-263. 


\section{2-286}

5. Dong J, Xu H, Xu H et al. Nesfatin-1 stimulates fatty-acid oxidation by activating AMP-activated protein kinase in STZ-induced type 2 diabetic mice. PLoS One 2013; 8: e83397.

6. Tang CH, Fu XJ, Xu XL, Wei XJ, Pan HS. The anti-inflammatory and antiapoptotic effects of nesfatin-1 in the traumatic rat brain. Peptides 2012; 36: 39-45.

7. Ari M, Ozturk OH, Bez Y, Oktar S, Erduran D. High plasma nesfatin-1 levelin patients with major depressive disorder. Prog Neuropsychopharmacol Biol Psychiatry 2011; 35: 497-500.

8. Aydin S, Dag E, Ozkan Y, Erman F, Dagli AF, Kilic N et al. Nesfatin-1 and ghrelin levels in serum and saliva of epileptic patients: hormonal changes can have amajor effect on seizure disorders. Mol Cell Biochem 2009; 328: 49-56.

9. Kuyumcu MS, Kuyumcu A, Yayla C et al., Nesfatin-1 levels in patients with slow coronary flow. Kardiol Pol 2018; 76 (2): 401-405. doi: 10.5603/KP.a2017.0210.

10. Feijoo-Bandin S, Rodriguez-Penas D, Garcia-Rua V, MosqueraLeal A, Gonzalez-Juanatey JR, Lago F. Nesfatin-1: a new energy-regulating peptide with pleiotropic functions. Impl Cardiovasc Level Endocr 2016; 52 (1): 11-29.

11. WHO Global atlas on cardiovascular disease prevention and control (2011). http://www.who.int/cardiovascular_diseases/publications/atlas_ cvd/en/. Accessed 15 July 2015.

12. Basak T, Varshney S, Akhtar S, Sengupta S. Understanding different facets of cardiovascular diseases based on model systems to human studies: a proteomic and metabolomic perspective. J Proteomics 2015. doi: 10.1016/j.jprot.2015.04.027.

13. Daniels SR. Prevention of atherosclerotic cardiovascular disease: what is the best approach and how early should we start? J Am Coll Cardiol 2014; 63: 2786-2788. doi: 10.1016/j.jacc.2014.01.082.

14. Hansson GK. Inflammation, atherosclerosis, and coronary artery disease. N Engl J Med 2005; 352: 1685-1695.
15. Hansson GK, Libby P, Tabas I. Inflammation and plaque vulnerability. J Intern Med 2015; 278: 483-493.

16. Yosten GL, Samson WK. Nesfatin-1 exerts cardiovascular actions in brain: possible interaction with the central melanocortin system. Amer J Physiol Regul Integr Comp Physiol 2009; 297 (2): R330-336.

17. Crea F, Liuzzo G. Pathogenesis of acute coronary syndromes. J Am Coll Cardiol 2013; 61 (1): 1-11. doi: 10.1016/j.jacc.2012.07.064.

18. Libby P. Inflammation in atherosclerosis. Nature 2002; 420 (6917) 868-874.

19. Dai H, Li X, He T et al. Decreased plasma nesfatin-1 levels in patients with acute myocardial infarction. Peptides 2013; 46: 167-171. doi: 10.1016/j.peptides.2013.06.006.

20. Kuyumcu A. The relationship between nesfatin-1 and carotid artery stenosis. Scand Cardiovasc J 2018; 52 (6): 328-334. doi: 10.1080/14017431.2018.1547840.

21. Ding S, Qu W, Dang S et al. Serum nesfatin-1 is reduced in type 2 diabetes mellitus patients with peripheral arterial disease. Med Sci Monit 2015; 21: 987-991. doi: 10.12659/MSM.892611.

22. Tasatargil A, Kuscu N, Dalaklioglu S et al. Cardioprotective effect of nesfatin-1 against isoproterenol-induced myocardial infarction in rats: Role of the Akt/GSK-3 $\beta$ pathway. Peptides 2017; 95: 1-9. doi: 10.1016/j. peptides.2017.07.003.

23. Li QC, Wang HY, Chen X, Guan HZ, Jiang ZY. Fasting plasma levels of nesfatin- 1 in patients with type 1 and type 2 diabetes mellitus and the nutrient-related fluctuation of nesfatin-1 level in normal humans. Regul Pept 2010; 159 (1-3): 72-77. doi: 10.1016/j.regpep.2009.11.003.

24. Zhai T, Li SZ, Fan XT, Tian Z, Lu XQ, Dong J. Circulating Nesfatin-1 Levels and Type 2 Diabetes: A Systematic Review and Meta-Analysis. J Diabetes Res 2017; 2017: 7687098. doi: 10.1155/2017/7687098.

25. Su Y, Zhang J, Tang Y, Bi F, Liu JN. The novel function of nesfatin-1: anti-hyperglycemia. Biochem Biophys Res Commun 2010; 391 (1): 1039-1042. doi: 10.1016/j.bbrc.2009.12.014. 